\title{
Multiaxial characterization of the mechanical behaviour of aluminium foam
}

\author{
L. Peroni, M. Avalle \& P. Martella \\ Department of Mechanics, \\ Politecnico di Torino (Technical University of Turin), Italy
}

\begin{abstract}
In the past ten years many new processes for making foamed metals, mostly aluminium or aluminium alloys, have been developed. As a matter of fact, closed-cell aluminium foam offers a unique combination of properties such as low density, high stiffness, strength, and energy absorption capability.

One of the main differences in the mechanical behaviour of cellular materials with respect to classical homogeneous materials such as solid metals is that foam failure is not independent from a hydrostatic state of stress. Therefore, it is not possible to describe the failure surface from a single, usually uniaxial, test but it is necessary to perform tests with different combination of deviatoric and hydrostatic stress components.

Within the European Project APROSYS, whose main objective is increasing the safety of all road-users, the mechanical behaviour of aluminium foam, under multiaxial loading, was investigated by the authors. In this paper the results of the hydrostatic and hydro-compression experimental tests are reported. From the results of these tests, it has been possible to obtain the yield locus of the aluminium foam in the deviatoric-hydrostatic stress components space.

Keywords: aluminium foam, hydrostatic, hydrocompression.
\end{abstract}

\section{Introduction}

It is well know that the mechanical behaviour of a cellular material, like foam, is not independent from a hydrostatic state of stress as, on the contrary, it happens for classical homogeneous materials such as metals. Therefore, it is not possible to describe the failure surface from a single simple uniaxial test (in tension or compression if there is a different behaviour) but it is necessary to perform tests 
with different combinations of deviatoric and hydrostatic stress components [1]. A test that gives pure deviatoric stress is, for example, a shear test. A test that produces pure hydrostatic stress in the material is the hydrostatic or triaxial test. A tensile test gives a combination of deviatoric and hydrostatic stress components.

In a material insensitive to the hydrostatic component of stress (as usually are considered metals) the failure limit in a deviatoric-hydrostatic space is a horizontal line. When the deviatoric stress reaches the yield line yielding occurs, therefore for metals it is possible to indefinitely increase the hydrostatic stress without yielding. If this hypothesis can be accepted a single experimental test gives the complete yield locus for this material.

The yield locus is different for foams (and other plastic materials) because there is an influence of the hydrostatic stress component [2-3]. A single experimental test is not enough to characterize the material behaviour completely. As a consequence a series of different tests are necessary in order to cover the widest possible range of combinations of deviatoric and hydrostatic stress components.

\section{Experimental tests}

Within the $6^{\text {th }}$ Framework Programme European project APROSYS [4], a complete characterisation of aluminium foam [5-8] was carried out at Politecnico di Torino. The experimental tests were performed in the Laboratory of the $2^{\text {nd }}$ Faculty of Engineering, in Vercelli. In this paper the hydrostatic compression and the hydro-compression tests are reported.
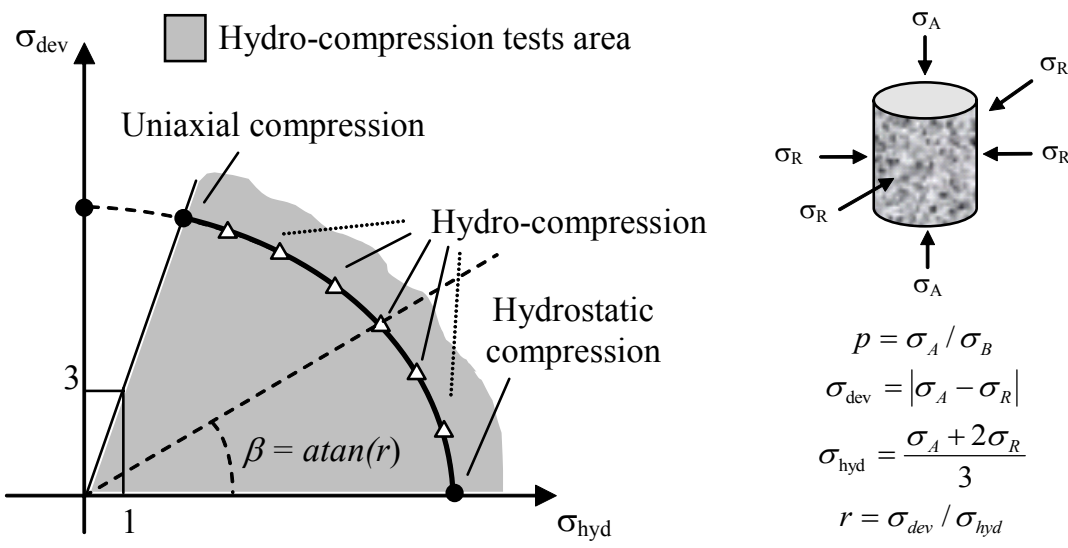

Figure 1:

Hydrostatic and hydro-compression tests area in the deviatorichydrostatic stress components space.

Hydrostatic compression tests were performed first to assess the behaviour of the tested aluminium foam in a pure hydrostatic stress condition. Then hydro- 
compression tests were carried out changing the ratio $p$ in order to obtain different $\sigma_{\text {hyd }}-\sigma_{\text {dev }}$ combinations. As shown in fig. 1, combining in different ways the axial stress $\sigma_{A}$ and the radial stress $\sigma_{R}$ it is possible to investigate the highlighted area of the $\sigma_{h y d}-\sigma_{d e v}$ plane. The limits of this area are the pure hydrostatic test $\left(\sigma_{A}=\sigma_{R}\right)$ and the pure compression test $\left(\sigma_{R}=0\right)$.

\subsection{Testing device}

Performing hydrostatic and hydro-compression tests on a metal foam is a challenging task. The foam specimen have to be completely separated from the fluid used to load it; for this reason it was covered with a latex sheath in order to avoid seepage of the fluid in the specimen. The cover have to be enough strong to not break during the test and, at the same time, it have to be very light and thin in order to not change the test results. Furthermore in the hydro-compression tests it is necessary to control the axial and the radial pressure separately. For all this reasons a testing device (fig. 2) was designed and built expressly to perform these tests. The test chamber, containing the foam specimen, was filled with a fluid (mixture of water and glycol) and mounted directly on the fixtures of a general purpose hydraulic testing machine DARTEC HA100 controlled with a DARTEC 9600 electronic unit.

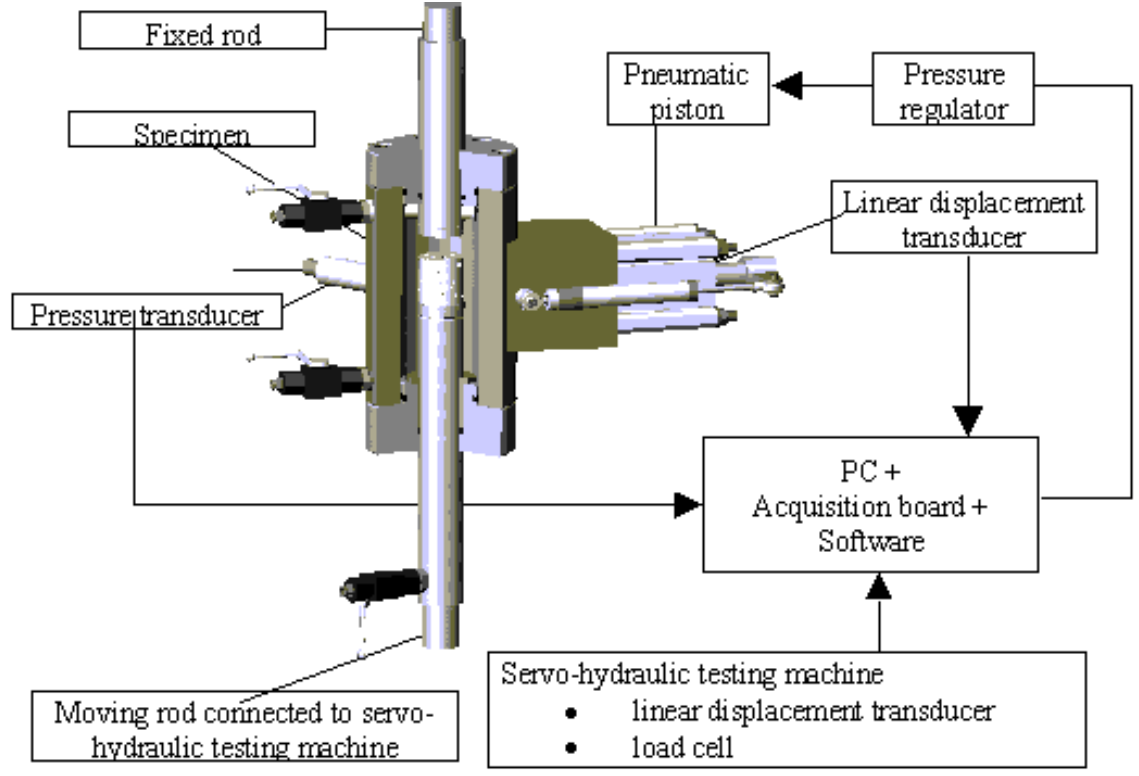

Figure 2: $\quad$ Schematic of the loading system.

In the hydrostatic tests the specimen was put on the vertical rod connected to the testing machine. This rod could move inside the test chamber in the axial direction changing the volume of the chamber and, consequently, the pressure of the fluid inside the chamber, i.e. the pressure on the specimen. 
In the hydro-compression tests the moving rod connected to the hydraulic testing machine pressed the specimen against a fixed rod inside the chamber generating the axial stress $\sigma_{A}$ on the specimen. Meantime, a double-effect pneumatic piston, was connected to the lateral surface of the test chamber. The rod of this piston could move inside the test chamber in the radial direction changing the volume of the chamber and, consequently, the pressure inside the chamber, generating the radial stress $\sigma_{R}$ on the specimen. In both tests the electronic unit performed the test control and the data acquisition, while a PC equipped with a National Instruments acquisition board was used to acquire the load, stroke and pressure. In the hydro-compression tests the PC carried out the control of the radial pressure $\sigma_{R}$ (pressure inside the test chamber) by moving the piston rod, whose displacement was measured through a potentiometer. The load applied though the rod connected to the testing machine was measured with a $100 \mathrm{kN}$, class A, strain-gage load cell, while the stroke of the rod was measured by means of a LVDT transducer connected to the hydraulic actuator. The pressure inside the chamber was measured with a 350 bar strain-gauge pressure transducer applied to the chamber.

\subsection{Samples}

The samples were cylinders cut from blocks, made of aluminium foam, produced by the Fraunhofer-Institute for Applied Materials Research (IFAM) in Bremen (D). These blocks were obtained through a powder metallurgical process for preparing foamed metals. According to this process, commercial powders are mixed with small quantities of a powdered foaming agent by means of conventional techniques. The mixture is compacted to a semi-finished product of low porosity by applying compaction techniques such as extrusion or coextrusion. The result of the compaction process is a foamable semi-finished product that can be worked into sheets, profiles, etc. by applying conventional deformation techniques. During a final heat treatment at temperatures above the melting point of the corresponding alloy, the material expands and develops its highly porous, closed-cell structure.

Three blocks $(308 \mathrm{~mm} \times 125 \mathrm{~mm} \times 45 \mathrm{~mm})$, identified as B2, B3 and B4, were cut in seven sub-blocks each to obtain the samples. These prismatic square section sub-blocks were turned with a $\mathrm{CNC}$ lathe so as to get circular cylindrical specimens with a nominal diameter and height of $41 \mathrm{~mm}$. As in the quasi-static compression tests (not reported in this paper) an anisotropic behaviour of the specimens was found, the specimens were machined in two different ways. In the ones obtained from block B2 the axial direction was parallel to the topbottom direction of the block (fig. 3). On the contrary, the specimens from blocks B3 and B4 the axial direction was parallel to the transversal direction of the block (fig. 3). In the foaming process the top-bottom direction is the first foaming direction (direction for which the boundary planes of the block were reached first), while the transverse and the longitudinal directions were the second and the third foaming direction respectively. 


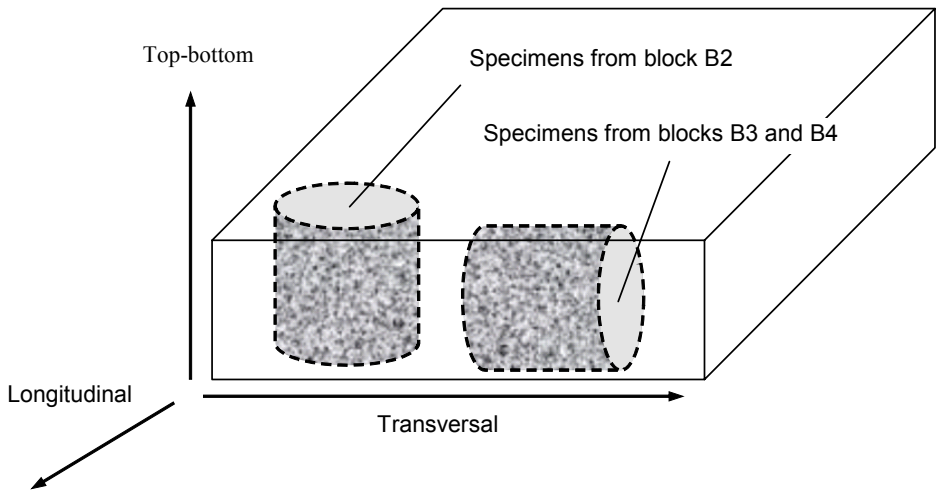

Figure 3: Position of the axis of the cylindrical specimens with respect to the block directions.

The properties of the specimens used for hydrostatic compression and hydrocompression tests are reported in the following tables.

Table 1: $\quad$ Properties of the specimens for hydrostatic compression tests.

\begin{tabular}{|c|c|c|c|c|}
\hline \multirow{2}{*}{$\begin{array}{c}\text { SPECIMEN } \\
\text { CODE }\end{array}$} & \multicolumn{2}{|c|}{ DIMENSIONS (mm) } & \multirow{2}{*}{$\begin{array}{c}\text { DENSITY } \\
\left(\mathbf{k g} / \mathbf{d m}^{\mathbf{3}} \mathbf{)}\right.\end{array}$} & $\begin{array}{c}\text { AXIAL } \\
\text { DIRECTION }\end{array}$ \\
\cline { 2 - 3 } & Diameter & Height & & Top-Bottom \\
\hline H-B2-1 & 41.2 & 41.8 & 0.66 & Top-Bottom \\
\hline H-B2-2 & 40.8 & 39.7 & 0.66 & Transversal \\
\hline H-B3-1 & 40.8 & 45.5 & 0.64 & Transversal \\
\hline H-B3-2 & 40.8 & 45.3 & 0.37 & Transversal \\
\hline H-B4-1 & 40.8 & 41.2 & 0.27 & Transversal \\
\hline H-B4-2 & 41.0 & 41.0 & 0.36 & Transversal \\
\hline H-B4-3 & 41.0 & 40.7 & 0.40 & \\
\hline
\end{tabular}

Table 2: $\quad$ Properties of the specimens for hydro-compression tests.

\begin{tabular}{|c|c|c|c|c|}
\hline \multirow{2}{*}{$\begin{array}{c}\text { SPECIMEN } \\
\text { CODE }\end{array}$} & \multicolumn{2}{|c|}{ DIMENSIONS (mm) } & \multirow{2}{\text{DENSITY}}{} & $\begin{array}{c}\text { SPECIMEN AXIAL } \\
\text { DIRECTION }\end{array}$ \\
\cline { 2 - 3 } & Diameter & Height & & Top-Bottom \\
\hline HC-B2-1 & 40.6 & 40.5 & 0.40 & Top-Bottom \\
\hline HC-B2-2 & 40.8 & 39.2 & 0.34 & Top-Bottom \\
\hline HC-B2-3 & 40.6 & 40.2 & 0.57 & Transversal \\
\hline HC-B3-1 & 40.8 & 45.3 & 0.54 & Transversal \\
\hline HC-B3-2 & 40.8 & 45.3 & 0.47 & Transversal \\
\hline HC-B3-3 & 40.8 & 45.0 & 0.38 & Transversal \\
\hline HC-B4-1 & 40.8 & 45.4 & 0.60 & \\
\hline
\end{tabular}




\section{Experimental results}

\subsection{Hydrostatic compression tests}

The results of the hydrostatic compression tests are reported in fig. 4. As it is possible to notice, the results are strongly dependent from the density which is the apparent density, the average value within the volume of the sample. Since there is a large scatter in size of the cells, cell thickness, and cell distribution the apparent density is affected by a very large scatter; as a consequence the test results are rather scattered. However a general behaviour can be detected. The elastic modulus, the yield stress and the slope of the stress-strain curve after yielding, all increase with increasing density.

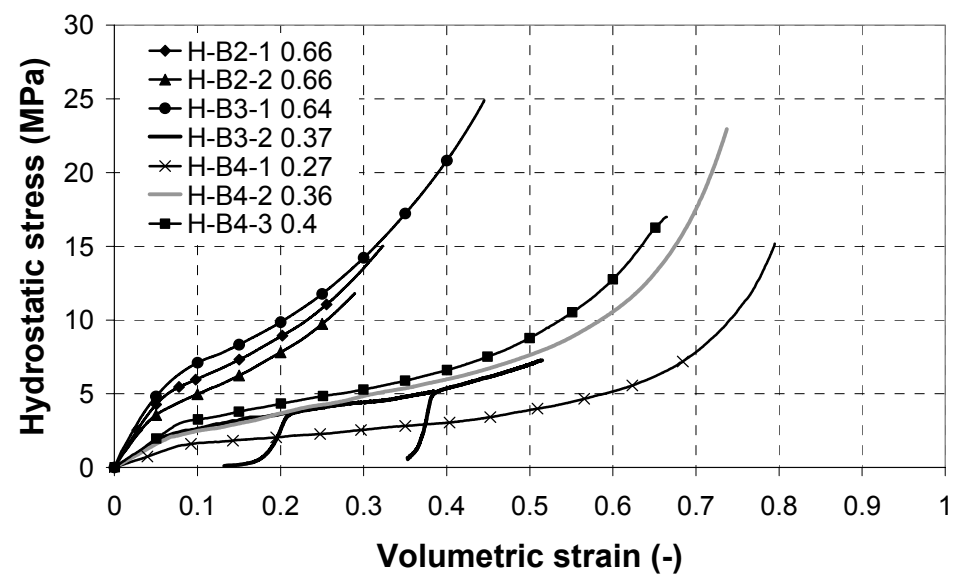

Figure 4: Hydrostatic compression tests: stress-strain curves

Furthermore, the tested foam showed an anisotropic behaviour, which can be detected by examining the deformed shapes of some specimens after the tests (fig 5). In specimen H-B3-1 the directions of the cross section are the longitudinal and the top-bottom directions of the base block. Because the topbottom direction is weaker than the longitudinal one, the cross section of the deformed specimen is elliptical (fig 5.(a)). This didn't occur in the H-B2-2 specimen for which the directions of the cross section are the longitudinal and the transversal directions of the base block, which have approximately the same strength. As a consequence, despite the slightly lower density, the yield stress of specimen H-B3-1 is higher than the one of the H-B2-1 and H-B2-2 specimens.

The hydrostatic yield stress for the specimens with a transversal axial direction was evaluated as intersection of the two lines interpolating the first part (elastic) and the second part (plateau) of the experimental stress-strain curve. An equation, which relates the hydrostatic yield stress to the density, was found through an interpolation of these experimental data.

$$
\sigma_{\text {yield-hyd }}=13.38 \cdot \rho^{1.773}
$$


Density $\rho$ in the previous equation is expressed in $\mathrm{kg} / \mathrm{m}^{3}$ and stress in $\mathrm{MPa}$.

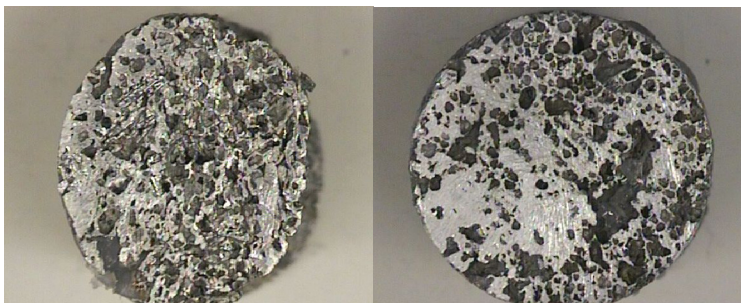

(a)

(b)

Figure 5: Cross section of H-B3-1 (a) and H-B2-2 (b) specimen after the tests.

In fig. 6 hydrostatic tests are compared with uniaxial quasi-static uniaxial tests (not reported in this paper) for two different densities. As it can be noticed the yield stress is higher for the uniaxial test because in this loading condition the specimen material is not constrained in the radial direction and therefore it can move laterally before the axial yield. Furthermore, the densification rate is greater for the hydrostatic loading condition.

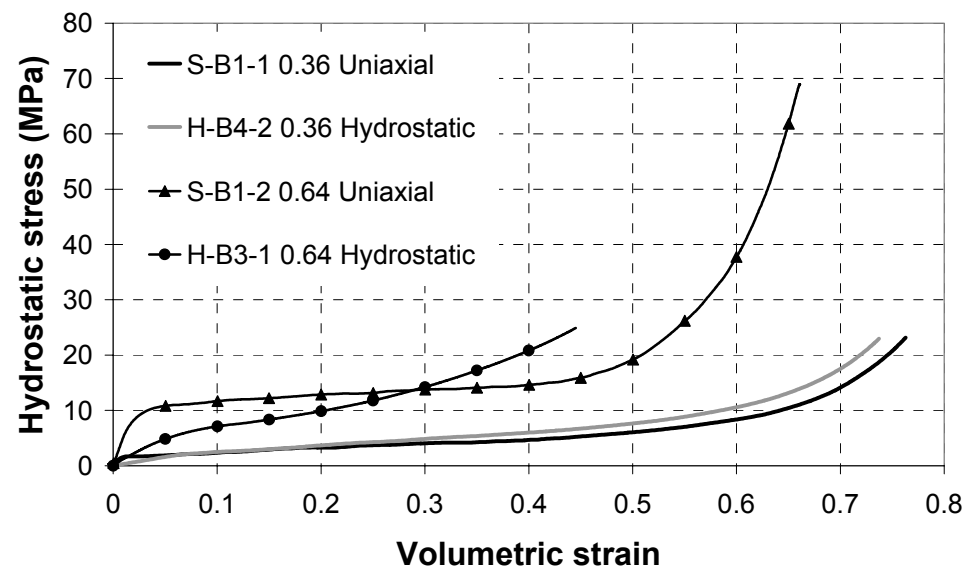

Figure 6: Comparison of uniaxial quasi-static tests and hydrostatic tests.

\subsection{Hydro-compression tests}

As explained above, one of the main aims of the experimental tests was evaluating the failure locus of the foam as shown in fig. 1. For this reason each hydro-compression test was performed applying a proportional load, i.e. a fixed ratio $\sigma_{R} / \sigma_{A}$, as shown in the following table. In addition two hydrostatic tests, 
on specimen H-B3-1 and H-B3-2 (reported in the previous section) were carried out using the testing device for the hydro-compression tests with a ratio $p=1$.

Table 3: $\quad$ Hydro-compression tests settings.

\begin{tabular}{|c|c|c|c|}
\hline SPECIMEN & $\begin{array}{l}\text { Density } \\
\left(\mathrm{kg} / \mathrm{m}^{3}\right)\end{array}$ & $p=\sigma_{R} / \sigma_{A}$ & $r=\sigma_{d e v} / \sigma_{h y d}$ \\
\hline HC-B2-1 & 0.40 & $p=0.658$ & $r=0.445$ \\
\hline HC-B2-2 & 0.34 & $p=0.366$ & $r=1.111$ \\
\hline HC-B2-3 & 0.57 & $p=0.658$ & $r=0.445$ \\
\hline HC-B3-1 & 0.54 & $p=0.658$ & $r=0.445$ \\
\hline HC-B3-2 & 0.47 & $p=0.366$ & $r=1.111$ \\
\hline HC-B3-3 & 0.38 & $p=0.658$ & $r=0.445$ \\
\hline HC-B4-1 & 0.60 & $p=0.366$ & $r=1.111$ \\
\hline H-B3-1 & 0.64 & $p=1$ (hydrostatic) & $r=0^{\circ}$ \\
\hline H-B3-2 & 0.37 & $p=1$ (hydrostatic) & $r=0^{\circ}$ \\
\hline
\end{tabular}

Examining the experimental data (axial and radial stress-strain curves), it was possible to notice a different behaviour in the axial and radial directions. This behaviour is shown clearly in the fig. 7 , where the results of the hydrostatic test performed on specimen H-B3-2 are reported. This test was performed with the experimental device for the hydro-compression tests so it was possible to evaluate the axial and the radial characteristics separately.

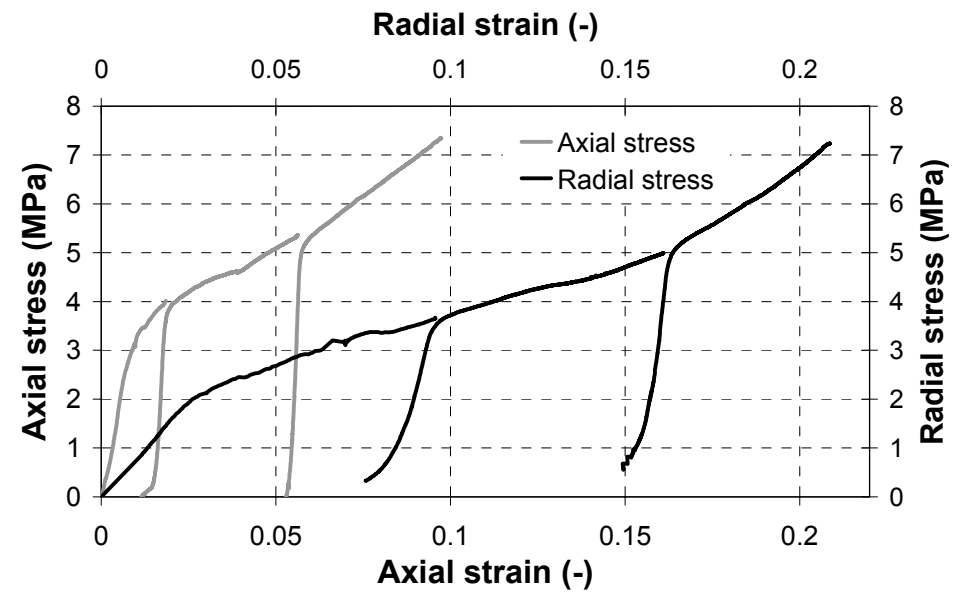

Figure 7: $\quad$ Hydrostatic test $(p=1)$ on specimen H-B3-2.

In fig. 7 , it is possible to notice that, even if the load ratio $p=1$ was kept at a constant level during the test, the axial and the radial yield stress were quite 
different. This means that the ratio between the axial yield stress and the radial yield stress was different from the ratio $p$ at which the experimental test was carried out. This event is rather clear in the hydrostatic tests, while in the hydrocompression tests it occurred only some times and not in a so evident way. However, due to this phenomenon, it was not possible to use the experimental data directly to obtain the failure locus because it was not clear which one of the yield stresses should have been chosen. In order to overcome this problem the following procedure was used. First of all, the hydrostatic stress-volumetric strain curve was calculated from the axial and radial stress-strain curves through the following equations:

$$
\sigma_{\text {hyd }}=\left(\sigma_{A}+2 \sigma_{R}\right) / 3, \varepsilon_{v o l}=\varepsilon_{A}+2 \varepsilon_{R}
$$

Then the yield stress for each test was evaluated considering the hydrostatic stress-volumetric strain curve. In order to remove the effect of the density on the results, each yield stress, calculated as described above, was divided by the theoretical yield stress evaluated through eqn (1). Therefore a normalised yield $\sigma_{\text {yield-adim }}$ stress was obtained

Finally, the normalised deviatoric and hydrostatic stress components were obtained combining the following equation:

$$
\sigma_{R}=p \sigma_{A}, \sigma_{h y d}=\left(\sigma_{A}+2 \sigma_{R}\right) / 3, \sigma_{d e v}=\left|\sigma_{A}-\sigma_{R}\right|
$$

These components are plotted in the $\sigma_{d e v}-\sigma_{h y d}$ (fig. 8) in order to obtain the failure locus.

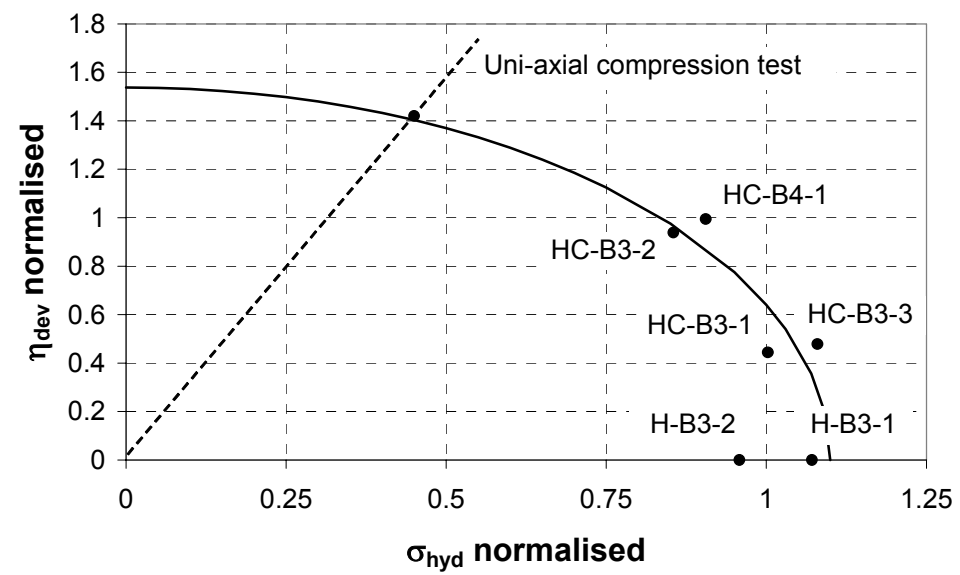

Figure 8: Failure locus. 


\section{Summary and conclusions}

Performing hydrostatic and hydro-compression tests on a metal foam proved to be a challenging and rather complicated task. The insulation of the specimen from the fluid used to load it was particularly difficult; indeed in many tests the cover used to protect the specimen from seepage of the fluid broke and the fluid penetrated into the specimen causing an early end of the test. For this reason many tests were carried out in order to set up the testing device and procedure; in this paper only the most important results are reported.

To sum up, the mechanical behaviour of aluminium foam was investigated through a series of experimental tests performed within the European Project APROSYS. As for foams the yielding is not independent from the hydrostatic stress component, a single experimental test is not enough to characterize the material behaviour completely. Therefore pure hydrostatic compression and hydro-compression tests were carried out through a testing device expressly developed. From the results of these tests it has been possible to obtain the yield locus of the aluminium foam in the deviatoric-hydrostatic stress components space.

\section{Acknowledgements}

The financial support of the European Commission by means of the APROSYS project and Mr. Dirk Lehmhus of Fraunhofer-Institute for Applied Materials Research (IFAM) are gratefully acknowledged.

\section{References}

[1] Gibson, L.J, Ashby, M.F., Cellular solids: structure and properties (Second Edition), Cambridge University Press, 1997.

[2] Collins, J.A., Failure of materials in mechanical design, John Wiley \& Sons, 1980.

[3] Khan, A.S., Huang, S., Continuum theory of plasticity, John Wiley \& Sons, 1995.

[4] Integrated Project on Advanced PROtection SYStems (APROSYS). On line: www.aprosys.com.

[5] Ashby, M.F., Evans, A., Fleck, N.A., Gibson, L.J., Hutchinson, J.W., Wadley, H.N.G., Metal foams - A Design Guide, Butterworth Heinemann, 2000.

[6] Ehlers, W., Mullerschon, H., Klar, O., On the behaviour of Aluminium Foams under Uniaxial and Multiaxial Loading, Verlag Mit, 1999.

[7] Deshpande, V.S., Fleck, N.A., Isotropic constitutive models for metallic foams, Journal of the Mechanics and Physics of Solids, Pergamon, 1999.

[8] Deshpande, V.S., Fleck, N.A., Multiaxial yield behaviour of polymer foams, Acta Materialia, Pergamon, 2001. 